\title{
Linear independence of linear forms in polylogarithms
}

\author{
RAFFAELE MARCOVECCHIO
}

\begin{abstract}
For $x \in \mathbb{C},|x|<1, s \in \mathbb{N}$, let $\operatorname{Li}_{s}(x)$ be the $s$-th polylogarithm of $x$. We prove that for any non-zero algebraic number $\alpha$ such that $|\alpha|<1$, the $\mathbb{Q}(\alpha)$-vector space spanned by $1, \operatorname{Li}_{1}(\alpha), \operatorname{Li}_{2}(\alpha), \ldots$ has infinite dimension. This result extends a previous one by Rivoal for rational $\alpha$. The main tool is a method introduced by Fischler and Rivoal, which shows the coefficients of the polylogarithms in the relevant series to be the unique solution of a suitable Padé approximation problem.
\end{abstract}

Mathematics Subject Classification (2000): 11J72 (primary); 11J17, 11J91, 33C20 (secondary).

\section{Introduction}

The aim of this paper is to prove the following statement: for $x \in \mathbb{C},|x|<1$, $s \in \mathbb{N}$, let $\operatorname{Li}_{s}(x)$ be the $s$-th polylogarithm of $x$ :

$$
\operatorname{Li}_{s}(x)=\sum_{k \geq 1} \frac{x^{k}}{k^{s}}
$$

then for any non-zero algebraic number $\alpha$ such that $|\alpha|<1$, in the sequence of complex numbers $1, \operatorname{Li}_{1}(\alpha), \operatorname{Li}_{2}(\alpha), \ldots$ infinitely many terms are linearly independent over the number field $\mathbb{Q}(\alpha)$.

More precisely, for any non-zero algebraic number $\alpha$ let $\mathrm{h}(\alpha)$ be the Weil absolute logarithmic height of $\alpha$ (see (4.7) below). Let $\delta(\alpha)=[\mathbb{Q}(\alpha): \mathbb{Q}]$ if $\alpha$ is real, and let $\delta(\alpha)=[\mathbb{Q}(\alpha): \mathbb{Q}] / 2$ otherwise. Let $\tau_{\alpha}(a)$ be the dimension of the $\mathbb{Q}(\alpha)$-vector space spanned by $1, \operatorname{Li}_{1}(\alpha), \ldots, \mathrm{Li}_{a}(\alpha)$.

We prove the following:

Theorem 1.1. For any $\varepsilon, \omega, H$ such that $\varepsilon, H>0$ and $0<\omega<1$, there exists an integer $\tilde{a}=\tilde{a}(\varepsilon, \omega, H)$ such that for every $a \geq \tilde{a}$ and for every non-zero algebraic

Received August 29, 2005; accepted in revised form November 17, 2005. 
number $\alpha$ with $|\alpha| \leq \omega$ and $\mathrm{h}(\alpha) \leq H$ the dimension $\tau_{\alpha}(a)$ satisfies

$$
\tau_{\alpha}(a) \geq \frac{1}{\delta(\alpha)} \cdot \frac{1-\varepsilon}{1+\log 2} \log a .
$$

In the special case of a non-zero rational number $\alpha$ such that $|\alpha|<1$, Rivoal [4] recently proved this result by applying the Nesterenko criterion to suitable linear forms in $1, \operatorname{Li}_{1}(\alpha), \ldots, \operatorname{Li}_{a}(\alpha)$ with rational coefficients. Rivoal's method is based on the series

$$
n !^{a-r} \sum_{k \geq 1} \frac{(k-1)(k-2) \cdots(k-r n)}{k^{a}(k+1)^{a} \cdots(k+n)^{a}} z^{-k}
$$

where $r=r(a)$ is an integer such that $1 \leq r<a, n \in \mathbb{N}$ and $z=\alpha^{-1}$. He mentioned that his result easily extends to real algebraic numbers $\alpha$, but for technical reasons the case of complex $\alpha$ is delicate. Indeed, Nesterenko's criterion needs both lower and upper bounds of the sequence of the linear forms as $n \rightarrow \infty$. Now, to obtain an asymptotic estimate of (1.2) is straightforward if $\alpha$ is real, but seems hard for non-real $\alpha$.

The method of [4] is inspired by Nikishin's series (see [3])

$$
\sum_{k \geq 1} \frac{(k-1)(k-2) \cdots(k-a n-q+1)}{k^{a} \cdots(k+n-1)^{a}(k+n)^{q}} z^{-k}, \quad q=0, \ldots, a .
$$

Using this tool, Nikishin proved that, for suitable negative rational numbers $\alpha$, all the real numbers $1, \operatorname{Li}_{1}(\alpha), \ldots, \operatorname{Li}_{a}(\alpha)$ are linearly independent over $\mathbb{Q}$. Each series (1.3) is easily seen to be a linear form in $1, \mathrm{Li}_{1}(1 / z), \ldots, \mathrm{Li}_{a}(1 / z)$ with polynomial coefficients lying in $\mathbb{Q}[z]$. The main feature of $(1.3)$ is that, for $q=0, \ldots, a$, the $a+1$ vectors of such polynomial coefficients are linearly independent. Thus, by a straightforward application of our Proposition 4.1 below, the use of the $a+1$ series (1.3) requires only upper bounds of the coefficients and of the linear forms, whereas the use of a single series such as (1.2) also requires a lower estimate of the corresponding linear form, and here is precisely where one would require a subtle application of the saddle point method. On the other hand, Nikishin's method essentially corresponds to the special case $r=a$ in Rivoal's series (1.2), so that, for fixed $a$, it applies only to a class of $\alpha$ 's smaller than Rivoal's.

In the present paper we employ a system of $a+1$ series that encompasses both Nikishin's and Rivoal's ideas:

$$
n !^{a-r} \sum_{k \geq 1} \frac{(k-r n)_{r n}}{(k)_{n}^{a}(k+n)^{q}} z^{-k}, \quad q=0, \ldots, a,
$$

where $(\beta)_{m}$ denotes the Pochhammer symbol:

$$
(\beta)_{0}=1, \quad(\beta)_{m}=\beta(\beta+1) \cdots(\beta+m-1) \quad(m=1,2, \ldots) .
$$


In Section 2 we state the upper bounds and the arithmetic properties of the linear forms and of the coefficients of (1.4).

The main new point of the present construction is to resume Nikishin's original argument, which does not need a precise asymptotic estimate of the sequence of linear forms. This is possible because a crucial property of (1.4) is the non-vanishing of the determinant of order $a+1$ of the coefficients of $1, \operatorname{Li}_{1}(\alpha), \ldots, \mathrm{Li}_{a}(\alpha)$, for $q=0,1, \ldots, a$ and $z=\alpha^{-1}$. To achieve our plan, in section 3 we resort to a recent method introduced by Fischler and Rivoal ([2], Theorem 1), which in particular shows that the vector $\left(P_{0}(z), \ldots, P_{a}(z)\right)$ of the coefficients of the polylogarithms in the series (1.2):

$$
n !^{a-r} \sum_{k \geq 1} \frac{(k-r n)_{r n}}{(k)_{n+1}^{a}} z^{-k}=\sum_{h=1}^{a} P_{h}(z) \mathrm{Li}_{h}(1 / z)-P_{0}(z)
$$

is the unique non-zero solution (up to a multiplicative constant) of the following Padé approximation problem:

$$
\begin{aligned}
& \sum_{h=1}^{a} P_{h}(z) \operatorname{Li}_{h}(1 / z)-P_{0}(z)=O\left(z^{-r n-1}\right) \quad(z \rightarrow \infty) \\
& \sum_{h=1}^{a} P_{h}(z) \frac{(\log (1 / z))^{h-1}}{(h-1) !}=O\left((1-z)^{a n+a-r n-1}\right) \quad(z \rightarrow 1) \\
& P_{0}(z), P_{1}(z), \ldots, P_{a}(z) \in \mathbb{C}[z], \operatorname{deg} P_{1}(z), \ldots, \operatorname{deg} P_{a}(z) \leq n .
\end{aligned}
$$

This property extends to the series (1.4) in a natural way (see our Remark 3.1 at the end of Section 3).

In the last section we complete the proof by using a suitable generalization of Nikishin's determinant argument.

We remark that the present extension from polylogarithms of rational numbers to polylogarithms of algebraic numbers is analogue to the extension made in [1], where Amoroso and Viola obtain good approximation measures for logarithms of algebraic numbers by generalizing a previous method of Viola [5] for logarithms of rational numbers.

Acknowledgements. I am grateful to Professor Amoroso for helpful discussions and interesting comments on this work. 


\section{Upper bounds}

Throughout this paper, $a, n, q, r$ are integers such that $0 \leq q \leq a, r=r(a)$ with $1 \leq r<a$ and $n \in \mathbb{N}$ (we let $n \rightarrow \infty$ ). Let also $D_{\lambda}=\frac{1}{\lambda !}\left(\frac{\mathrm{d}}{\mathrm{d} t}\right)^{\lambda}$, and let

$$
R_{n}(k)=n !^{a-r} \frac{(k-r n)_{r n}}{(k)_{n}^{a}(k+n)^{q}}
$$

for chosen $a, q, r$.

In this section, following closely the methods of [3] and [4], we write each series $(1.4)$ as a linear form in $1, \operatorname{Li}_{1}(1 / z), \ldots, \mathrm{Li}_{a}(1 / z)$ with polynomial coefficients, and we derive the required upper bounds and arithmetic properties of the linear forms and of the coefficients.

For any $a, n, q, r$ as above, and for any complex number $z$ with $|z|>1$, let $N_{n}(a, q, r ; z)$ be the series (1.4):

$$
N_{n}(a, q, r ; z)=\sum_{k \geq 1} R_{n}(k) z^{-k} .
$$

It is worth noting that $N_{n}(a, q, r ; z)$ is a generalized hypergeometric function of $z^{-1}$ :

$$
\begin{aligned}
& N_{n}(a, q, r ; z)=\frac{n !^{a-r}(r n) !^{a+1}}{((r+1) n) !^{a-q}((r+1) n+1) !^{q}} z^{-r n-1} \times \\
& \quad \times \sum_{s \geq 0} \frac{(r n+1)_{s}^{a+1}}{((r+1) n+1)_{s}^{a-q}((r+1) n+2)_{s}^{q}} \frac{z^{-s}}{s !} .
\end{aligned}
$$

We denote (for fixed $r$ and $a$ )

$c_{h, q, j, n}= \begin{cases}D_{a-h}\left(R_{n}(t)(t+j)^{a}\right)_{\mid t=-j} \in \mathbb{Q} & \text { if } j=0, \ldots, n-1 ; h=1, \ldots, a \\ D_{q-h}\left(R_{n}(t)(t+n)^{q}\right)_{\mid t=-n} \in \mathbb{Q} & \text { if } j=n ; h=1, \ldots, q \\ 0 & \text { if } j=n ; h=q+1, \ldots, a\end{cases}$

The rational function $R_{n}(t)$ decomposes as follows:

$$
R_{n}(t)=\sum_{h=1}^{a} \sum_{j=0}^{n} \frac{c_{h, q, j, n}}{(t+j)^{h}} .
$$

We now define the following polynomials in $z$ :

$$
\begin{aligned}
A_{n}^{(h)}(a, q, r ; z) & =\sum_{j=0}^{n} c_{h, q, j, n} z^{j} \quad(h=1, \ldots, a) \\
B_{n}(a, q, r ; z) & =\sum_{h=1}^{a} \sum_{j=1}^{n} c_{h, q, j, n} \sum_{k=1}^{j} \frac{1}{k^{h}} z^{j-k} .
\end{aligned}
$$


For the forthcoming analysis it is useful to remark that the degree of $A_{n}^{(h)}(a, q, r ; z)$ does not exceed $n$ for $1 \leq h \leq q$, and does not exceed $n-1$ for $q+1 \leq h \leq a$. Moreover, for any $h=1, \ldots, a$ the degree of $A_{n}^{(h)}(a, h, r ; z)$ is exactly $n$, since

$$
0 \neq c_{h, h, n, n}=\left(R_{n}(t)(t+n)^{h}\right)_{\mid t=-n}= \pm \frac{((r+1) n) !}{n !^{r+1}} .
$$

We also notice that the coefficient of the power $z^{-t}$ in $N_{n}(a, q, r ; z)$ is zero for $t=1, \ldots, r n$, and non-zero for all $t \geq r n+1$. In addition, the coefficient of $z^{-r n-1}$ in $N_{n}(a, 0, r ; z)$ is

$$
\frac{n !^{a-r}(r n) !^{a+1}}{((r+1) n) !^{a}} .
$$

Using the decomposition (2.2) in the formula (2.1), one readily sees that

$$
N_{n}(a, q, r ; z)=\sum_{h=1}^{a} A_{n}^{(h)}(a, q, r ; z) \operatorname{Li}_{h}(1 / z)-B_{n}(a, q, r ; z)
$$

The next three Lemmas 2.1, 2.2 and 2.3 are very similar to Lemmas 3, 4 and 5 of [4], and to Lemmas 1, 2 and 3 of [3], so we omit the proofs.

Lemma 2.1. For all $z \in \mathbb{C}$ with $|z|>1$

$$
\limsup _{n \rightarrow \infty}\left|N_{n}(a, q, r ; z)\right|^{1 / n} \leq \frac{1}{|z|^{r} r^{a-r}} .
$$

Lemma 2.2. For all $z \in \mathbb{C}$ we have

$$
\begin{aligned}
& \limsup _{n \rightarrow \infty}\left|A_{n}^{(h)}(a, q, r ; z)\right|^{1 / n} \leq r^{r} 2^{a+r+1} \max \{1,|z|\} \quad(h=1, \ldots, a), \\
& \limsup _{n \rightarrow \infty}\left|B_{n}(a, q, r ; z)\right|^{1 / n} \leq r^{r} 2^{a+r+1} \max \{1,|z|\} .
\end{aligned}
$$

Lemma 2.3. Let $d_{n}$ be the least common multiple of the integers $1, \ldots, n$.

Then

$$
\begin{aligned}
d_{n}^{a-h} A_{n}^{(h)}(a, q, r ; z) & \in \mathbb{Z}[z] \quad(h=1, \ldots, a), \\
d_{n}^{a} B_{n}(a, q, r ; z) & \in \mathbb{Z}[z] .
\end{aligned}
$$

\section{A non-vanishing determinant}

To shorten our notation, from now on we put $A_{n}^{(h)}(q)=A_{n}^{(h)}(a, q, r ; z), B_{n}(q)=$ $B_{n}(a, q, r ; z), N_{n}(q)=N_{n}(a, q, r ; z)$. 
Let $M_{n}$ be the following square matrix of order $a+1$ :

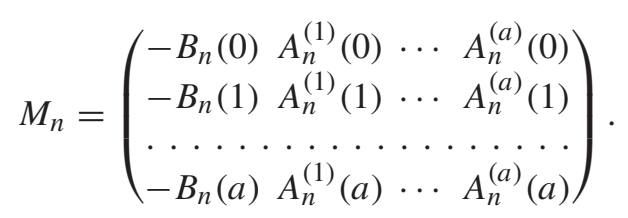

We claim that for any complex number $z \neq 1$ the matrix $M_{n}$ is non-singular.

Clearly, $\operatorname{det}\left(M_{n}\right)$ is a polynomial in $z$. On the other hand, for any complex number $z$ such that $|z|>1$, we can add to the first column of $M_{n}$ a linear combination of the other columns, namely we can replace $-B_{n}(0)$ with the sum $-B_{n}(0)+$ $A_{n}^{(1)}(0) \operatorname{Li}_{1}(1 / z)+\cdots+A_{n}^{(a)}(0) \operatorname{Li}_{a}(1 / z)$ in the first row of $(3.1),-B_{n}(1)$ with $-B_{n}(1)+A_{n}^{(1)}(1) \operatorname{Li}_{1}(1 / z)+\cdots+A_{n}^{(a)}(1) \operatorname{Li}_{a}(1 / z)$ in the second row of (3.1), and so on, and by applying (2.5) we immediately see that

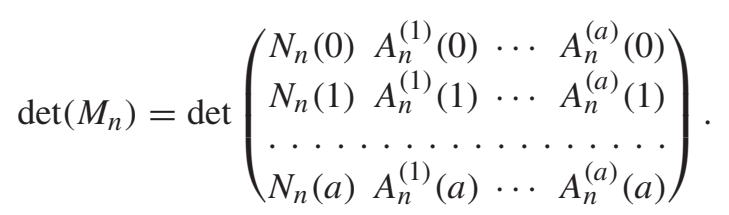

We now recall that for $t=1, \ldots, r n$ the coefficient of $z^{-t}$ is zero in each of $N_{n}(0), \ldots, N_{n}(a)$, and $\operatorname{deg} A_{n}^{(h)}(q) \leq n$ for $h=1, \ldots, a$ and for $q=0, \ldots, a$. Therefore, expanding the determinant on the right hand side of (3.2) along the first column, we obtain

$$
\operatorname{det}\left(M_{n}\right)=z^{a n-r n-1} \sum_{k \geq 0} u_{k} z^{-k}
$$

for suitable rational numbers $u_{k}$. Moreover, since the elements above the principal diagonal of the matrix in (3.2) satisfy $\operatorname{deg} A_{n}^{(h)}(q) \leq n-1(h=q+1, \ldots, a)$ and for the elements of that diagonal $\operatorname{deg} A_{n}^{(h)}(h)=n(h=1, \ldots, a)$, the coefficient $u_{0}$ is the product of the coefficient of $z^{-r n-1}$ in $N_{n}(0)$ and of the leading coefficients of the polynomials $A_{n}^{(1)}(1), \ldots, A_{n}^{(a)}(a)$. Thus

$$
0 \neq u_{0}= \pm\left(\frac{(r n) !}{n !^{r}}\right)^{a+1}
$$

by (2.3) and (2.4). Since $z$ is arbitrary in the domain $|z|>1$, from the previous analysis we infer that the degree of the polynomial $\operatorname{det}\left(M_{n}\right)$ is exactly $a n-r n-1$, and that its leading coefficient is $u_{0}$.

Following Fischler and Rivoal [2], we now introduce, for $z \notin]-\infty, 0$ ], the function

$$
J_{n}(a, q, r ; z)=\frac{1}{2 \pi i} \oint_{|t|=\mu n} R_{n}(t) z^{-t} d t
$$


$(i=\sqrt{-1})$ where $\mu>1$ is arbitrary. As above, we abbreviate $J_{n}(q)=J_{n}(a, q, r ; z)$. Using the decomposition (2.2) and Cauchy's integral formula we obtain

$$
J_{n}(q)=A_{n}^{(1)}(q)+\sum_{h=2}^{a} A_{n}^{(h)}(q) \frac{\left(\log \frac{1}{z}\right)^{h-1}}{(h-1) !} .
$$

Furthermore,

$$
\left(\frac{\mathrm{d}^{k}}{\mathrm{~d} z^{k}} J_{n}(a, q, r ; z)\right)_{\mid z=1}=\frac{(-1)^{k}}{2 \pi i} \oint_{|t|=\mu n} n !^{a-r} \frac{(t-r n)_{r n}}{(t)_{n}^{a}(t+n)^{q}}(t)_{k} d t,
$$

and letting $\mu \rightarrow \infty$ we see that $J_{n}(q)$ vanishes at $z=1$ to the order $a n+q-$ $r n-1$. As before, we can add to the second column of (3.1) a linear combination of the subsequent columns, namely we can replace $A_{n}^{(1)}(0)$ with the sum $A_{n}^{(1)}(0)+$ $A_{n}^{(2)}(0) \frac{\log \frac{1}{z}}{1 !}+\cdots+A_{n}^{(a)}(0) \frac{\left(\log \frac{1}{z}\right)^{a-1}}{(a-1) !}$ in the first row of (3.1), $A_{n}^{(1)}(1)$ with $A_{n}^{(1)}(1)+$ $A_{n}^{(2)}(1) \frac{\log \frac{1}{z}}{1 !}+\cdots+A_{n}^{(a)}(1) \frac{\left(\log \frac{1}{z}\right)^{a-1}}{(a-1) !}$ in the second row of (3.1), and so on, and by using (3.4) we obtain

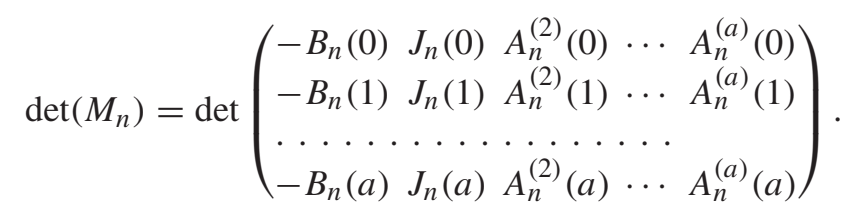

Thus, expanding the determinant on the right hand side of (3.5) along the second column, we see that $\operatorname{det}\left(M_{n}\right)$ vanishes at $z=1$ with multiplicity at least $a n-r n-1$. Since $\operatorname{deg}\left(M_{n}\right)=a n-r n-1$, we must have

$$
\operatorname{det}\left(M_{n}\right)= \pm \frac{(r n) !^{a+1}(z-1)^{a n-r n-1}}{n !^{r(a+1)}} .
$$

In particular, for any $z \neq 1$ this implies that

$$
\operatorname{det}\left(M_{n}\right) \neq 0
$$

as we claimed.

Remark 3.1. As is noted in [2, page 1383], by generalizing (1.5) one can prove that the so-called non-diagonal Padé approximation problem

$$
\begin{aligned}
& \sum_{h=1}^{a} p_{h}(z) \operatorname{Li}_{h}(1 / z)-p_{0}(z)=O\left(z^{-r n-1}\right) \quad(z \rightarrow \infty) \\
& \sum_{h=1}^{a} p_{h}(z) \frac{(\log (1 / z))^{h-1}}{(h-1) !}=O\left((1-z)^{a n+q-r n-1}\right) \quad(z \rightarrow 1) \\
& p_{0}(z), p_{1}(z), \ldots, p_{a}(z) \in \mathbb{C}[z] \\
& \operatorname{deg} p_{1}(z), \ldots, \operatorname{deg} p_{q}(z) \leq n, \quad \operatorname{deg} p_{q+1}(z), \ldots, \operatorname{deg} p_{a}(z) \leq n-1
\end{aligned}
$$


has a unique solution (up to a multiplicative constant), namely $p_{0}(z)=B_{n}(q)$, $p_{h}(z)=A_{n}^{(h)}(q)(h=1, \ldots, a)$.

\section{Generalization of the Nikishin determinant method}

Let $\mathbb{K} \subset \mathbb{C}$ be a number field. Let $\mathcal{M}_{\mathbb{K}}$ be the set of places of $\mathbb{K}$, and let Id $\in \mathcal{M}_{\mathbb{K}}$ be the archimedean place associated with the usual absolute value in $\mathbb{C}$. For any $v \in \mathcal{M}_{\mathbb{K}}$, let $\mathbb{K}_{v}$ be the completion of $\mathbb{K}$ with respect to $v$, and let $\eta_{v}=\left[\mathbb{K}_{v}\right.$ : $\mathbb{Q}_{v}$ ]. Thus, $\eta_{\mathrm{Id}}=1$ if $\mathbb{K} \subset \mathbb{R}$, and $\eta_{\mathrm{Id}}=2$ otherwise. Let $|\cdot|_{v}$ be the absolute value associated with $v$, normalized as follows: if $v \mid \infty$ and $v$ is associated with an embedding $\sigma: \mathbb{K} \rightarrow \mathbb{C}$, we denote $|\beta|_{v}=|\sigma(\beta)|$ for $\beta \in \mathbb{K}$; if, instead, $v \mid p$, where $p$ is a rational prime, we let $|p|_{v}=1 / p$. We also put $\delta=[\mathbb{K}: \mathbb{Q}] / \eta_{\text {Id }}$.

For $\boldsymbol{\beta}=\left(\beta_{1}, \ldots, \beta_{m}\right) \in \mathbb{K}^{m}$ we define

$$
\mathrm{h}_{0}(\boldsymbol{\beta})=\frac{1}{[\mathbb{K}: \mathbb{Q}]} \sum_{\substack{v \in \mathcal{M}_{\mathbb{K}} \\ v \neq \mathrm{Id}}} \eta_{v} \log |\boldsymbol{\beta}|_{v},
$$

where $|\boldsymbol{\beta}|_{v}=\max \left\{\left|\beta_{1}\right|_{v}, \ldots,\left|\beta_{m}\right|_{v}\right\}$. We remark that $\mathrm{h}_{0}(\boldsymbol{\beta})$ depends only on $\boldsymbol{\beta}$, and is independent of the field $\mathbb{K}$.

We need the following:

Proposition 4.1. Let $\boldsymbol{\theta}=\left(\theta_{1}, \ldots, \theta_{m}\right) \in\left(\mathbb{C}^{\times}\right)^{m}$, let

$$
\mathcal{L}_{i}^{(n)}(\boldsymbol{x})=l_{i, 1}^{(n)} x_{1}+\cdots+l_{i, m}^{(n)} x_{m} \quad(i=1, \ldots, m ; n \in \mathbb{N})
$$

be linear forms with coefficients in a number field $\mathbb{K} \subset \mathbb{C}$, and suppose that for any $n \in \mathbb{N}$ the linear forms $\mathcal{L}_{1}^{(n)}, \ldots, \mathcal{L}_{m}^{(n)}$ are linearly independent.

Assume also that $($ for $i=1, \ldots, m)$

$$
\begin{aligned}
& \limsup _{n \rightarrow \infty} \frac{1}{n} \log \left|\mathcal{L}_{i}^{(n)}(\boldsymbol{\theta})\right| \leq-\rho \\
& \limsup _{n \rightarrow \infty} \frac{1}{n} \log \max \left\{\left|l_{i, 1}^{(n)}\right|, \ldots,\left|l_{i, m}^{(n)}\right|\right\} \leq c \\
& \limsup _{n \rightarrow \infty} \frac{1}{n} \mathrm{~h}_{0}\left(\boldsymbol{L}_{i}^{(n)}\right) \leq c^{\prime},
\end{aligned}
$$

where $\boldsymbol{L}_{i}^{(n)}=\left(l_{i, 1}^{(n)}, \ldots, l_{i, m}^{(n)}\right)$, and $\rho, c, c^{\prime}$ are real numbers satisfying $c, c^{\prime}, \rho+c>0$.

Then the dimension $\tau$ of the $\mathbb{K}$-vector space spanned by $\theta_{1}, \ldots, \theta_{m}$ satisfies

$$
\tau \geq \frac{c+\rho}{c+\delta c^{\prime}}
$$


Proof. Let

$$
A=\left(\begin{array}{ccc}
a_{\tau+1,1} & \cdots & a_{\tau+1, m} \\
\cdots & \cdots & \ldots \\
a_{m, 1} & \cdots & a_{m, m}
\end{array}\right)
$$

be a matrix with entries in $\mathbb{K}$, having $m-\tau$ linearly independent rows such that

$$
a_{i, 1} \theta_{1}+\cdots+a_{i, m} \theta_{m}=0 \quad(i=\tau+1, \ldots, m) .
$$

Then for each $n$, up to renumbering $\boldsymbol{L}_{1}^{(n)}, \ldots, \boldsymbol{L}_{m}^{(n)}$, we can assume that the square matrix

$$
\Lambda^{(n)}=\left(\begin{array}{ccc}
l_{1,1}^{(n)} & \cdots & l_{1, m}^{(n)} \\
\cdots & \cdots & \cdots \\
l_{\tau, 1}^{(n)} & \cdots & l_{\tau, m}^{(n)} \\
a_{\tau+1,1} & \cdots & a_{\tau+1, m} \\
\cdots & \cdots & \cdots \\
a_{m, 1} & \cdots & a_{m, m}
\end{array}\right)
$$

is non-singular. On multiplying by $\theta_{1}$ the first column of $\Lambda^{(n)}$ and using (4.1) and (4.4) we get

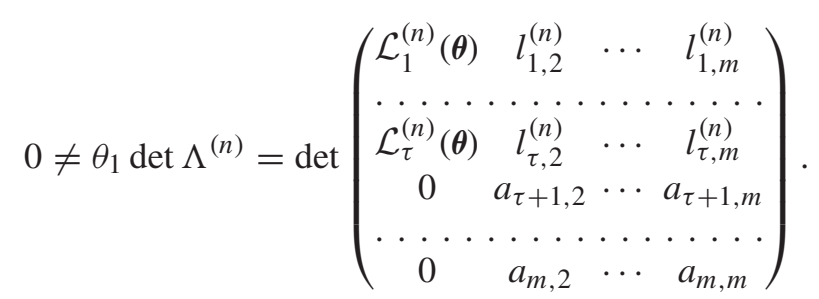

We now apply the product formula to $\theta_{1} \operatorname{det} \Lambda^{(n)}$, and for each place $v \in \mathcal{M}_{\mathbb{K}}$ we use either (4.5) or (4.6), according as $v \neq \mathrm{Id}$ or $v=\mathrm{Id}$, respectively. We have

$$
\begin{aligned}
0= & \eta_{\mathrm{Id}} \log \left|\theta_{1} \operatorname{det} \Lambda^{(n)}\right|+\sum_{\substack{v \in \mathcal{M}_{\mathbb{K}} \\
v \neq \mathrm{Id}}} \eta_{v} \log \left|\theta_{1} \operatorname{det} \Lambda^{(n)}\right|_{v} \\
\leq & \eta_{\mathrm{Id}}\left(\log \max _{1 \leq i \leq \tau}\left|\mathcal{L}_{i}^{(n)}(\boldsymbol{\theta})\right|+(\tau-1) \log \max _{\substack{1 \leq i \leq \tau \\
2 \leq j \leq m}}\left|l_{i, j}^{(n)}\right|\right) \\
& +\tau \sum_{\substack{v \in \mathcal{M}_{\mathbb{K}} \\
v \neq \mathrm{Id}}} \eta_{v} \log \max _{\substack{1 \leq i \leq \tau \\
1 \leq j \leq m}}\left|l_{i, j}^{(n)}\right|_{v}+\gamma,
\end{aligned}
$$

for some real number $\gamma$ which is independent of $n$. Dividing by $\eta_{\text {Id }} \times n$ and letting $n \rightarrow \infty$, from (4.2) we obtain $0 \leq-\rho+(\tau-1) c+\tau \delta c^{\prime}$, which is the same as (4.3). 
We are ready to prove Theorem 1.1. Before doing this we recall the definition of the Weil absolute logarithmic height:

$$
\mathrm{h}(\beta)=\frac{1}{[\mathbb{K}: \mathbb{Q}]} \sum_{v \in \mathcal{M}_{\mathbb{K}}} \eta_{v} \log ^{+}|\beta|_{v} \quad(\beta \in \mathbb{K}) .
$$

Here and in the sequel $\log ^{+} x=\log \max \{x, 1\}$ for $x \geq 0$. It is well known that $\mathrm{h}(\beta)$ depends only on $\beta$, and is independent of the field $\mathbb{K}$.

Theorem 1.1 now follows from a straightforward application of the following analogue of Proposition 1 of [4]:

Proposition 4.2. Let $a, r$ be integers such that $1 \leq r<a$, and let $\alpha \in \overline{\mathbb{Q}}^{\times}$with $|\alpha|<1$. Then

$$
\tau_{\alpha}(a) \geq \frac{1}{\delta(\alpha)} \cdot \frac{a \log r+(a+r+1) \log 2-(r+1) \log |\alpha|}{a+(a+r+1) \log 2+r \log r+\mathrm{h}(\alpha)} .
$$

Proof. Let $q \in[0, a]$ be an integer. We apply Proposition 4.1 to the linear forms

$$
d_{n}^{a} N_{n}(q)=d_{n}^{a} A_{n}^{(1)}(q) \operatorname{Li}_{1}(\alpha)+\cdots+d_{n}^{a} A_{n}^{(a)}(q) \operatorname{Li}_{a}(\alpha)-d_{n}^{a} B_{n}(q),
$$

with $\mathbb{K}=\mathbb{Q}(\alpha), \delta=\delta(\alpha)$ and $z=\alpha^{-1}$. The Prime Number Theorem implies that $\lim _{n \rightarrow \infty} \frac{\log d_{n}}{n}=1$. Then by Lemma 2.1 we have

$$
\limsup _{n \rightarrow \infty} \frac{1}{n} \log \left|d_{n}^{a} N_{n}(q)\right| \leq a-(a-r) \log r+r \log |\alpha|=-\rho .
$$

Moreover, for all places $v \in \mathcal{M}_{\mathbb{K}}$ such that $v \mid \infty$, by Lemma 2 we get

$$
\begin{aligned}
\limsup _{n \rightarrow \infty} \frac{1}{n} \log \max \left\{\left|d_{n}^{a} B_{n}(q)\right|_{v},\left|d_{n}^{a} A_{n}^{(1)}(q)\right|_{v}, \ldots,\left|d_{n}^{a} A_{n}^{(a)}(q)\right|_{v}\right\} \\
\leq a+r \log r+(a+r+1) \log 2+\log ^{+}|z|_{v}=c_{v} .
\end{aligned}
$$

Let $c=c_{\mathrm{Id}}$, and note that $\rho+c>0$. Finally, Lemma 2.3 gives

$$
\max \left\{\left|d_{n}^{a} B_{n}(q)\right|_{v},\left|d_{n}^{a} A_{n}^{(1)}(q)\right|_{v}, \ldots,\left|d_{n}^{a} A_{n}^{(a)}(q)\right|_{v}\right\} \leq\left(\max \left\{1,|z|_{v}\right\}\right)^{n}
$$

for all places $v \in \mathcal{M}_{\mathbb{K}}$ such that $v \nmid \infty$. Hence

$$
\begin{aligned}
& \limsup _{n \rightarrow \infty} \frac{1}{n} \mathrm{~h}_{0}\left(d_{n}^{a} B_{n}(q), d_{n}^{a} A_{n}^{(1)}(q), \ldots, d_{n}^{a} A_{n}^{(1)}(q)\right) \\
& \leq \frac{1}{[\mathbb{K}: \mathbb{Q}]}\left(\sum_{\substack{v \mid \infty \\
v \neq \mathrm{Id}}} \eta_{v} c_{v}+\sum_{v \nmid \infty} \eta_{v} \log ^{+}|z|_{v}\right) \\
& =a+r \log r+(a+r+1) \log 2+\mathrm{h}(z)-\frac{c}{\delta(\alpha)}=c^{\prime} .
\end{aligned}
$$


As for $c^{\prime}$, we remark that $\mathrm{h}(z)=\mathrm{h}\left(z^{-1}\right)=\mathrm{h}(\alpha)$. By (3.6) the linear forms $d_{n}^{a} N_{n}(0), \ldots, d_{n}^{a} N_{n}(a)$ are linearly independent.

From Proposition 4.1 we get (4.8).

Proof of Theorem 1.1. Just as in [4], let $r$ be the integer nearest to $a(\log a)^{-2}$. From Proposition 4.2 we have

$$
\begin{aligned}
& a \log r+(a+r+1) \log 2-(r+1) \log |\alpha|=a \log a+o(a \log a) \\
& a+(a+r+1) \log 2+r \log r+\mathrm{h}(\alpha)=(1+\log 2) a+o(a),
\end{aligned} \quad(a \rightarrow \infty),
$$

which implies (1.1) for all $a \geq \tilde{a}$, where $\tilde{a}$ depends only on $\varepsilon,|\alpha|$ and $\mathrm{h}(\alpha)$.

\section{References}

[1] F. AmOROSO and C. ViOLA, Approximation measures for logarithms of algebraic numbers, Ann. Scuola Norm. Sup. Pisa Cl. Sci. (4) 30 (2001), 225-249.

[2] S. Fischler and T. Rivonl, Approximants de Padé et séries hypergéométriques équilibrées, J. Math. Pures Appl. (9) 82 (2003), 1369-1394.

[3] E. M. Nikishin, On the irrationality of the values of the functions $F(x, s)$, Math. Sb. (N.S.) 109 (151) (1979), 410-417 (in Russian); English translation in Math. URSS-Sb. 37 (1980), 381-388.

[4] T. RivonL, Indepéndance linéaire de valeurs des polylogarithmes, J. Théor. Nombres Bordeaux 15 (2003), 551-559.

[5] C. Viola, Hypergeometric functions and irrationality measures, In: "Analytic Number Theory”, Y. Motohashi (ed.), London Math. Soc. Lecture Note Series 247, Cambridge Univ. Press, 1997, 353-360.

Dipartimento di Matematica Università di Pisa

Largo Bruno Pontecorvo, 5 56127 Pisa, Italy marcovec@mail.dm.unipi.it 